(2)

\title{
Radiotherapy for one rectal cancer patient with cirrhosis and moderate to severe thrombocytopenia: a case report
}

This article was published in the following Dove Press journal:

OncoTargets and Therapy

\author{
Xinqi Shi \\ Huifang Xia \\ Weiwei Zhang \\ Guang Li \\ Ailin Li
}

Department of Radiation Oncology, The First Hospital of China Medical University, Shenyang, Liaoning, China
Correspondence: Ailin Li

Department of Radiation Oncology,

The First Hospital of China Medical

University, I 55 Nanjingbei Street,

Shenyang I I000I, China

Tel +86 I 5840175046

Email liailin0312@।63.com

\begin{abstract}
When patients with cirrhosis and severe thrombocytopenia suffer malignant tumors, there is usually no effective and feasible treatment method due to the high risk of hemorrhage. Herein, we report a case in which radiotherapy was given to a patient with a strong desire for the treatment. The patient was a 66-year-old man with a 13-year history of cirrhosis and a 10 -year history of thrombocytopenia, and was diagnosed with locally advanced rectal cancer (LARC; $\mathrm{T}_{4 \mathrm{a}} \mathrm{N}_{1} \mathrm{M}_{0}$, stage IIIB). The platelet count before radiotherapy was $32 \times 10^{9} / \mathrm{L}$, and the blood coagulation was normal. The severity of thrombocytopenia increased after 2 Gy $\times 7$ fractions pelvic radiation, with platelet counts dropping to $16 \times 10^{9} / \mathrm{L}$. Platelet counts failed to return to pre-therapy levels after supporting therapy including recombinant human interleukin 11 subcutaneous injection and platelet transfusion. Although radiotherapy was discontinued eventually, the data presented here represent a valuable resource that can help inform treatment decisions for tumor patients with cirrhosis and thrombocytopenia.
\end{abstract}

Keywords: rectal cancer, liver cirrhosis, thrombocytopenia, radiotherapy

\section{Introduction}

When patients with post-hepatitis cirrhosis and severe thrombocytopenia suffer malignant tumors, there is usually no effective and feasible treatment method, such as surgical treatment, chemotherapy, or targeted treatment, due to the high risk of hemorrhage. To our knowledge, currently, there is no published literature on whether radiotherapy can be attempted in such a situation. Usually, when the platelet count of a malignant tumor patient is below $70 \times 10^{9} / \mathrm{L}$, radiotherapy is not considered because of the risk of bleeding. However, some researchers have argued that platelet count is not an effective predictor of unprovoked bleeding in cirrhotic patients. ${ }^{1}$ We report here a case of locally advanced rectal cancer (LARC) in a patient with a 13-year history of liver cirrhosis and a 10-year history of thrombocytopenia and leukopenia. As the patient and his family had a strong desire to undergo treatment, and no other viable methods are currently available, we attempted radiotherapy.

\section{Case report}

The patient was a 66-year-old man who was diagnosed with LARC in the upper middle rectum $\left(\mathrm{T}_{4 \mathrm{a}} \mathrm{N}_{1} \mathrm{M}_{0}\right.$, stage IIIB). He had a 13-year history of liver cirrhosis after contracting hepatitis B and exhibited symptoms of hypersplenism, including thrombocytopenia and leukopenia. No spontaneous bleeding events had happened in the past 10 years. Given the high risk of severe complications, radical surgery, chemotherapy, or targeted 
therapy could not be performed. However, the patient and his family had a strong desire for treatment and came to our radiotherapy department in October 2017, requesting that radiotherapy be attempted.

The test results before radiotherapy were as follows: A routine blood test of the patient on October 26, 2017, showed that his platelet count was $32 \times 10^{9} / \mathrm{L}$, white blood cell (WBC) count was $3.79 \times 10^{9} / \mathrm{L}$, and red blood cell (RBC) count was $3.57 \times 10^{12} / \mathrm{L}$; meanwhile, blood coagulation function was in the normal range (prothrombin time [PT], 13.7 seconds; activated partial thromboplastin time [APTT], 39.1 seconds; thrombin time [TT], 16.60 seconds; international normalized ratio [INR], 1.06). His hepatitis $B$ was in a stable condition $(\mathrm{HBsAg}(+), \operatorname{HBsAb}(-), \operatorname{HBeAg}(-)$, $\mathrm{HBe} \mathrm{Ab}(-)$, and $\mathrm{HBcAb}(+))$, and no hepatitis $\mathrm{C}$ virus (HCV) infection was detected $(\mathrm{HCVAb}(-))$. Liver function was Child-Pugh class A (no hepatic encephalopathy; no ascites; total bilirubin [TBIL], $21.9 \mu \mathrm{mol} / \mathrm{L}$; albumin (ALB), $38.9 \mathrm{~g} / \mathrm{L}$; PT prolong, $<3$ seconds). An ultrasound test of the liver revealed a normal size with rough surface and blunt border; intrahepatic echogenicity was enhanced, with scattering echoless points in the right lobe; and the hepatic vein was circuitous, with a rough wall. The volume of the spleen was abnormally large: $13.8 \mathrm{~cm}$ in length and $5.3 \mathrm{~cm}$ in thickness. An imaging examination of the patient showed no bone metastasis of the tumor. Moreover, the patient had shown no symptoms of spontaneous bleeding, such as petechiae or errhysis, in the past 10 years. The doctors involved had a comprehensive discussion about the patient's condition with his family, informing them that blood transfusions and supporting therapy would be required and that there would be a risk involved in the process of radiotherapy. A routine blood test on October 30, 2017, showed that his platelet count was $32 \times 10^{9} / \mathrm{L}$, WBC count was $4.03 \times 10^{9} / \mathrm{L}$, and $\mathrm{RBC}$ count was $3.65 \times 10^{12} / \mathrm{L}$. A pelvic-abdominal computed tomography (CT) simulation with intravenous injection contrast was obtained in the supine position. The high-risk clinical target volume (CTV-HR) included the entire mesorectum and presacral region at involved levels, with a 2-cm sup/inf margin on the gross tumor volume (GTV). The standard risk CTV (CTV-SR) covered the elective node subsites, depending on the tumor stage and location, including presacral space, mesorectum, posterior lateral lymph nodes, and anterior lateral lymph nodes of this patient. The planning target volume (PTV) of CTV-SR is generated by three-dimensional expanding CTV-SR with 1-cm margin plus in the sup/inf directions and $0.7-\mathrm{cm}$ margin plus in the anterior/posterior/right/left directions. Volumetric modulated arc therapy (VMAT) radiation with 50 Gy in 25 fractions was planned for PTV delivery. After that, a CTV-HR boost above 54 Gy (final total dose) was planned since the patient was unable to undergo surgery. The CTV-HR and CTV-SR are shown in Figure 1. The patient started the first treatment on October 31, 2017. We paid close attention to platelet count changes during the treatment process. On November 3 , 2017 , his platelet count was $30 \times 10^{9} / \mathrm{L}\left(\mathrm{WBC}, 3.38 \times 10^{9} / \mathrm{L}\right.$; $\left.\mathrm{RBC}, 3.48 \times 10^{9} / \mathrm{L}\right)$, and this had decreased to $25 \times 10^{9} / \mathrm{L}$ on November 7, 2017 (WBC, $3.31 \times 10^{9} / \mathrm{L}$; RBC, $\left.3.46 \times 10^{9} / \mathrm{L}\right)$. Since the platelet count decline was obvious, we recommended the injection of thrombopoietin (TPO; 3Sbio Inc., Shenyang, Liaoning, China). However, the cost of this treatment was prohibitively high for the patient. Taking into account these factors, we instead chose subcutaneous injections of $3 \mathrm{mg}$ recombinant human interleukin 11 (IL-11; Qilu Pharmaceutical, Jinan, Shandong, China) per day, starting on November 7, 2017, and continuing for 5 days. On November 9,2017 , the platelet count decreased to $16 \times 10^{9} / \mathrm{L}, \mathrm{WBC}$ count was $3.08 \times 10^{9} / \mathrm{L}, \mathrm{RBC}$ count was $2.99 \times 10^{12} / \mathrm{L}$, and the patient showed no signs of abnormal bleeding. At that time, he had received 2 Gy $\times 7$ fractions of radiotherapy on PTV (from October 31, 2017, to November 3, 2017, and from November 6, 2017, to November 8, 2017), and the treatment was discontinued. He then underwent $1 \mathrm{U}$ platelet transfusions on November 9 and 11, 2017. After that, his platelet count was $27 \times 10^{9} / \mathrm{L}$ on November 10,2017 , and $19 \times 10^{9} / \mathrm{L}$ on November 11,2017 . The number stabilized at $24-25 \times 10^{9} / \mathrm{L}$ during the following 5 days, but decreased to $22 \times 10^{9} / \mathrm{L}$ on November 17,2017 , and then remained at about $20 \times 10^{9} / \mathrm{L}$ for 3 days and failed to reach the previous levels. The counts of WBC and RBC showed no significant changes. Blood cell changes during treatment are shown in Figure 2. The patient and his family were informed that the radiotherapy might be continued if his platelet count could be maintained by platelet transfusion, but they finally decided to abandon the treatment.

A written informed consent has been provided by this patient to have the case details published.

\section{Discussion}

Thrombocytopenia is a common complication in patients with liver cirrhosis and is defined as a platelet count $<150 \times 10^{9} / \mathrm{L}$; $50-100 \times 10^{9} / \mathrm{L}$ is classified as moderate and $<50 \times 10^{9} / \mathrm{L}$ is classified as severe. ${ }^{2,3}$ At present, splenic platelet sequestration is considered to be the major cause of thrombocytopenia 

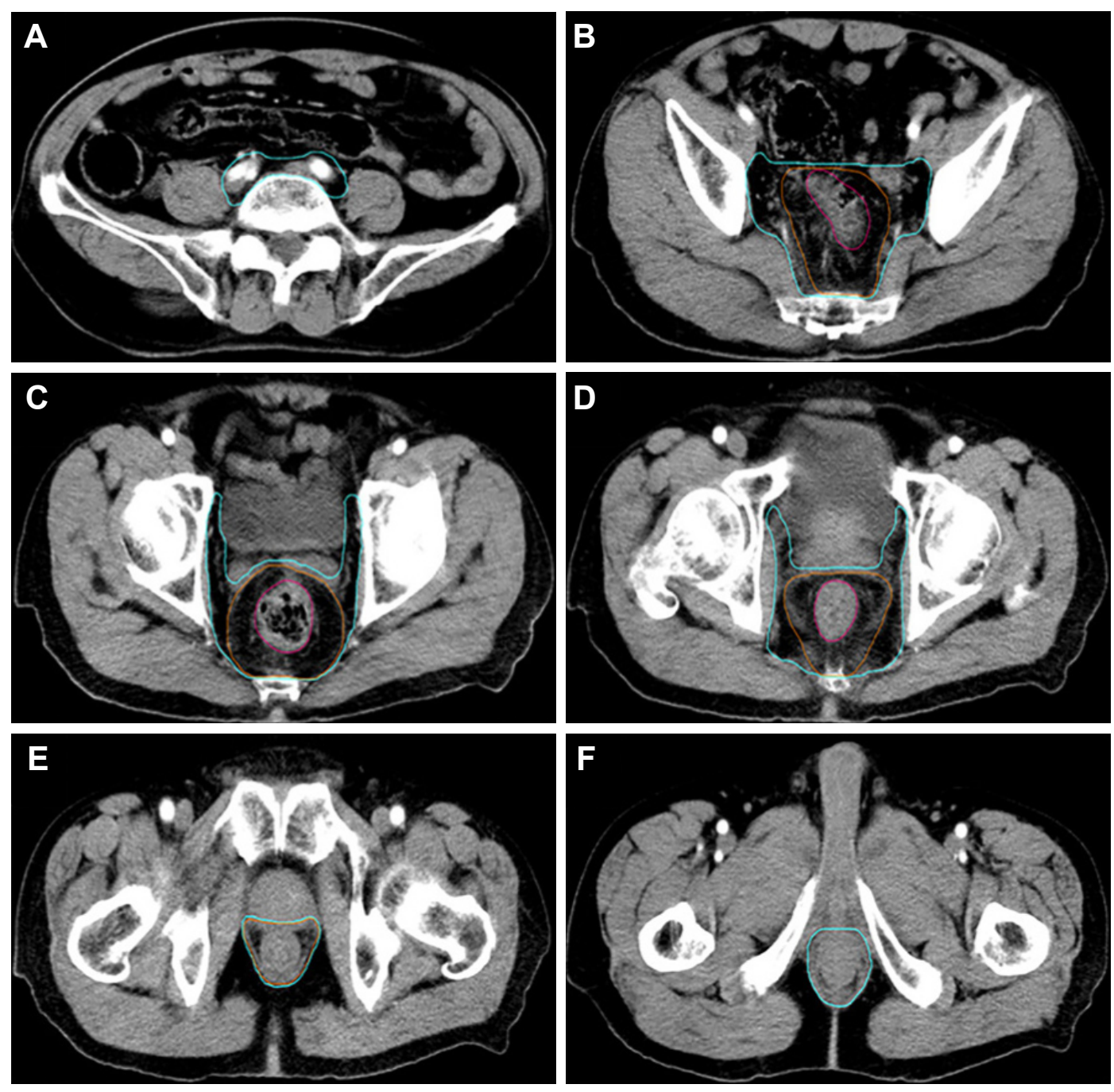

Figure I CTV-SR (blue), CTV-HR (orange), and GTV (red) on the simulation CT.

Notes: (A) The CT layer of the upper edge of CTV-SR; combined CTV-SR included PS and LLN-A. (B) The CT layer of the upper edge (rectosigmoid) of GTV; combined CTV-SR included PS, LLN-A, and M; CTV-HR included M around GTV. (C, D) The CT layers of mid-low (C) and low (D) pelvic; combined CTV-SR included PS, posterior LLN-P, LLN-A, and M; CTV-HR included M around GTV. (E, F) The CT layers of the lower edge of CTV-HR (E) and CTV-SR (F); CTV-SR and CTV-HR included M.

Abbreviations: CT, computed tomography; CTV-HR, high-risk clinical target volume; CTV-SR, standard risk clinical target volume; GTV, gross tumor volume; LLN-A, anterior lateral lymph nodes; LLN-P, lateral lymph nodes; M, mesorectum; PS, presacral space.

in cirrhotic patients, ${ }^{4-6}$ while immunological processes, chronic HCV infection, and a decrease in hematopoietic growth factors can also contribute to it. ${ }^{7}$ Studies have also shown that bone marrow function is suppressed in advanced cirrhosis and the potential for regeneration declines, and this contributes significantly to hematological abnormalities in patients. ${ }^{8}$ Thrombocytopenia is considered a major risk factor for bleeding events; however, in recent years, some researchers have argued that a "rebalance" would be established in patients with chronic liver disease ${ }^{9}$ as coagulation inhibiting factors decrease concomitantly when clotting factors decline. ${ }^{10}$ Although the balance could easily be destroyed by infections or other precipitating factors, these patients had less tendency to bleed than was previously thought. ${ }^{11}$ Spontaneous bleeding is not likely to occur until the platelet count is less than $10-20 \times 10^{9} / \mathrm{L}^{3,12}$

Myelosuppression is one of the major complications of radiotherapy and can result in decreased numbers of WBCs, platelets, and RBCs. Despite the cytotoxicity to human bone marrow cells and peripheral blood cells, most tumor patients can tolerate radiotherapy well in clinical practice. ${ }^{13}$ But in the case of the patient described in this study, platelet count declined much more sharply than generally observed and failed to improve after platelet transfusion and supportive treatment. While input platelets are likely to have been destroyed in the spleen, decreased bone marrow compensatory function due to 
A

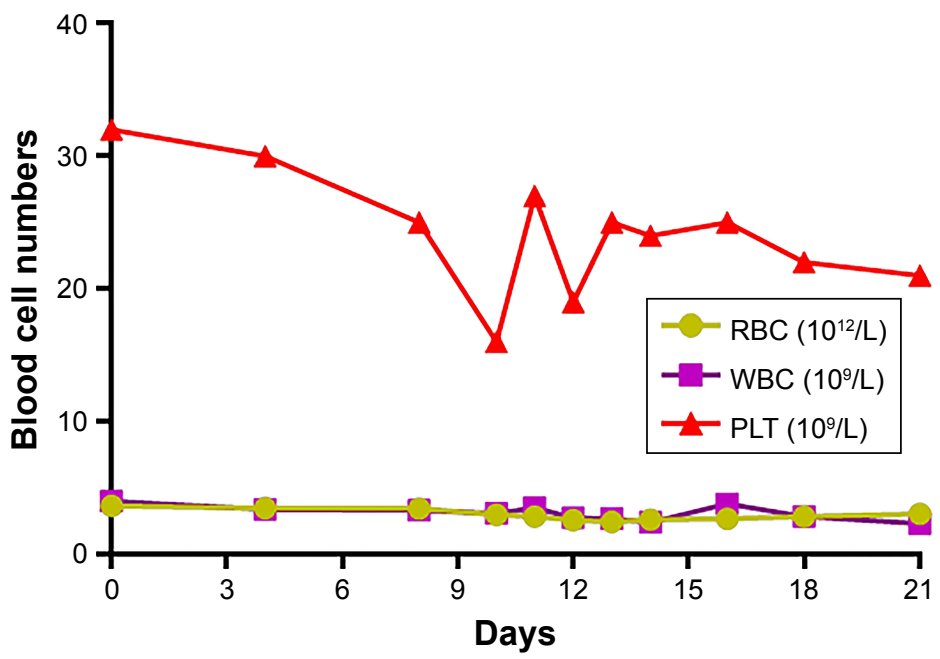

B

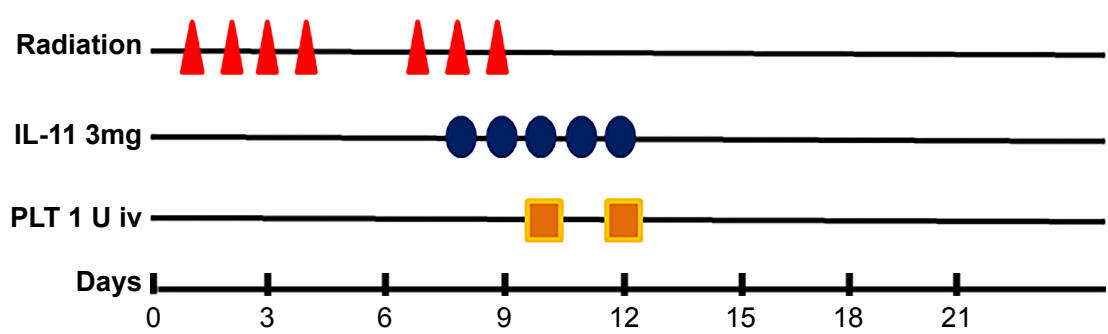

C

\begin{tabular}{llllllllllll}
\hline Days & 0 & 4th & 8th & 10th & 11th & 12th & 13th & 14th & 16th & 18th & 21st \\
\hline RBC $\left(10^{12} / \mathrm{L}\right)$ & 3.65 & 3.48 & 3.46 & 2.99 & 2.86 & 2.59 & 2.45 & 2.61 & 2.69 & 2.84 & 3.05 \\
WBC $\left(10^{9} / \mathrm{L}\right)$ & 4.03 & 3.38 & 3.31 & 3.08 & 3.50 & 2.74 & 2.68 & 2.42 & 3.80 & 2.84 & 2.32 \\
PLT $\left(10^{9} / \mathrm{L}\right)$ & 32 & 30 & 25 & 16 & 27 & 19 & 25 & 24 & 25 & 22 & 21 \\
\hline
\end{tabular}

Figure 2 The changes in complete blood count (RBC, WBC, and PLT) depended on the date and treatments (radiation, IL-II, and PLT infusion).

Notes: (A) The changes in RBC, WBC, and PLT depended on the date (days 0-2I). (B) 2 Gy radiation on PTV was delivered on days I-4 and days 7-9. Three milligrams of IL-II was subcutaneously injected on days 8-12. I U PLT was infused on days 10 and I2. (C) The numbers of RBC, WBC, and PLT on different time points.

Abbreviations: IL, interleukin; iv, intravenous; PLT, platelet; PTV, planning target volume; RBC, red blood cell; WBC, white blood cell.

long-term cirrhosis and thrombocytopenia must also be considered as this may lead to deficient hematopoiesis and poorer tolerance of radiotherapy. ${ }^{8}$ Therefore, for patients with liver cirrhosis and thrombocytopenia, bone marrow function may also be defective in addition to hypersplenism. Platelet transfusions are unlikely to be helpful for hypersplenism because the additional platelets would be sequestered in the spleen, but they may improve the thrombocytopenia caused by imperfect bone marrow function. ${ }^{14}$ We postulated that the patient was likely to complete the planned course of radiotherapy if platelets were continually transfused, but unfortunately we could not proceed with the planned treatment.

The patient had a 13-year history of cirrhosis and a 10-year history of thrombocytopenia, and hence, his bone marrow function was likely to have been adversely affected during this time and manifested as poor tolerance to radiotherapy. However, for patients with less severe or lengthy medical complications, tolerance to radiotherapy might be higher. Meanwhile, platelet transfusions can help maintain platelet count. In general, patients with cirrhosis and thrombocytopenia have relatively poorer bone marrow tolerance compared with other cancer patients; but, in spite of this, there are possibilities for them to undergo radiotherapy. Meanwhile, our experience also implies that if the patient does not receive continuous platelet input during radiotherapy, radiation therapy is not recommended.

\section{Conclusion}

There are possibilities for tumor patients with cirrhosis and thrombocytopenia to undergo radiotherapy, provided that the patient can accept continuous platelet infusions during radiotherapy.

\section{Acknowledgment}

This work was supported by the National Natural Science Foundation of China (no 81402520). 


\section{Disclosure}

The authors report no conflicts of interest in this work.

\section{References}

1. Basili S, Raparelli V, Napoleone L, et al. Platelet count does not predict bleeding in cirrhotic patients: results from the PRO-LIVER study. Am J Gastroenterol. 2018;113(3):368-375.

2. Buckley MF, James JW, Brown DE, et al. A novel approach to the assessment of variations in the human platelet count. Thromb Haemost. 2000;83(3):480-484.

3. Peck-Radosavljevic M. Thrombocytopenia in chronic liver disease. Liver Int. 2017;37(6):778-793.

4. Nowatari T, Murata S, Fukunaga K, Ohkohchi N. Role of platelets in chronic liver disease and acute liver injury. Hepatol Res. 2014;44(2): $165-172$.

5. Rauber P, Lammert F, Grotemeyer K, Appenrodt B. Immature platelet fraction and thrombopoietin in patients with liver cirrhosis: A cohort study. PLoS One. 2018;13(2):e0192271.

6. Lv Y, Yee Lau W, Wu H, et al. Causes of peripheral cytopenia in hepatitic cirrhosis and portal hypertensive splenomegaly. Exp Biol Med. 2017;242(7):744-749.
7. Gangireddy VG, Kanneganti PC, Sridhar S, Talla S, Coleman T. Management of thrombocytopenia in advanced liver disease. Can $J$ Gastroenterol Hepatol. 2014;28(10):558-564.

8. Bihari C, Anand L, Rooge S, et al. Bone marrow stem cells and their niche components are adversely affected in advanced cirrhosis of the liver. Hepatology. 2016;64(4):1273-1288.

9. Lisman T, Porte RJ. Rebalanced hemostasis in patients with liver disease: evidence and clinical consequences. Blood. 2010;116(6):878-885.

10. Ditisheim S, Goossens N, Spahr L, Hadengue A. Coagulation and cirrhosis: new insight. Revue medicale suisse. 2012;81652(352):1654-1656.

11. Leonardi F, Maria N, Villa E. Anticoagulation in cirrhosis: a new paradigm? Clin Mol Hepatol. 2017;23(1):13-21.

12. George JN. Platelets. Lancet (London, England). 2000;355(9214): 1531-1539.

13. Heylmann D, Rödel F, Kindler T, Kaina B. Radiation sensitivity of human and murine peripheral blood lymphocytes, stem and progenitor cells. Biochim Biophys Acta. 2014;1846(1):121-129.

14. Sekhon SS, Roy V. Thrombocytopenia in adults: a practical approach to evaluation and management. South Med J. 2006;99(5):491-498.

\section{Publish your work in this journal}

OncoTargets and Therapy is an international, peer-reviewed, open access journal focusing on the pathological basis of all cancers, potential targets for therapy and treatment protocols employed to improve the management of cancer patients. The journal also focuses on the impact of management programs and new therapeutic agents and protocols on

\section{Dovepress}

patient perspectives such as quality of life, adherence and satisfaction. The manuscript management system is completely online and includes a very quick and fair peer-review system, which is all easy to use. Visit http://www.dovepress.com/testimonials.php to read real quotes from published authors. 